\title{
Association of Interleukin-12B Gene Polymorphism With Multiple Sclerosis in Patients From Southeast of Iran
}

\author{
Maryam Nemati, ${ }^{1}$ Hossain-Ali Ebrahimi, ${ }^{2}$ Hossain Hajghani, ${ }^{3}$ Arezoo Khosravimashizi, ${ }^{3}$ and Abdollah \\ Jafarzadeh ${ }^{3,4, *}$ \\ ${ }^{1}$ Department of Laboratory Sciences, Paramedical School, Kerman University of Medical Sciences, Kerman, IR Iran \\ ${ }^{2}$ Neurology Research Center, Department of Neurology, Kerman University of Medical Sciences, Kerman, IR Iran \\ ${ }^{3}$ Department of Immunology, Medical School, Kerman University of Medical Sciences, Kerman, IR Iran \\ ${ }^{4}$ Molecular Medicine Research Center, Rafsanjan University of Medical Sciences, Rafsanjan, IR Iran \\ "Corresponding author: Abdollah Jafarzadeh, Professor of Immunology, Department of Immunology, Medical School, Kerman University of Medical Sciences, Kerman, Iran. \\ Tel: +98-3433221662, Fax: +98-3433221660, E-mail: Jafarzadeh14@yahoo.com
}

Received 2017 October 28; Accepted 2018 January 06.

\section{Abstract}

Background: The presence of polymorphisms in IL-12B gene, encoding IL-12 P40 subunit, is associated with a number of autoimmune diseases.

Objectives: The current study aimed at evaluating the association of a single nucleotide polymorphism (SNP; rs3212227) in IL-12B gene with multiple sclerosis (MS) in patients from Southeast of Iran.

Methods: Blood specimens were collected from140 patients with MS as the case and 140 gender-and age-matched healthy subjects as the control groups. The genomic DNA was extracted and the genetic variations in SNP rs3212227 were determined using polymerase chain reaction-restriction fragment length polymorphism (PCR-RFLP) technique.

Results: The frequencies of AA, AC, and CC genotypes in SNP rs3212227 were 55.0\%, 17.9\%, and 27.1\% in patients with MS, and 61.4\%, $29.3 \%$, and $9.3 \%$, in healthy subjects, respectively. The frequency of CC genotype at rs3212227 was significantly higher in patients with MS as compared with the healthy controls $(\mathrm{P}<0.001)$. However, the AC genotype was less prevalent in patients with MS than the healthy control ones $(\mathrm{P}<0.02)$. The frequency of A and C alleles at SNP rs3212227 were $63.09 \%$ and $36.07 \%$ in patients with MS and 76.07\% and 23.9\% in healthy control subjects, respectively. The frequency of $C$ allele was significantly higher, whereas the frequency of A allele was lower in patients with MS than the healthy control subjects $(\mathrm{P}<0.001)$. The frequency of CC genotype in SNP rs3212227 was significantly higher in patients with relapsing remitting MS (RRMS), secondary progressive MS (SPMS), and primary progressive MS (PPMS) patterns in comparison with the healthy control subjects $(\mathrm{P}<0.002, \mathrm{P}<0.001$ and $\mathrm{P}<0.05$, respectively). In patients with RRMS pattern, the frequency of AC genotype (18.6\%) was significantly lower than that of the control group $(29.3 \%, \mathrm{P}<0.05)$. In patients with RRMS and SPMS patterns the frequency of $C$ allele was significantly higher in comparison with that of the control group $(\mathrm{P}<0.03$ and $\mathrm{P}<0.001$, respectively).

Conclusions: The results represented that the presence of CC genotype and C allele in SNP rs3212227 of IL-12B gene were associated with susceptibility to MS disease, whereas the presence of AC genotype and A allele may confer protection against the disease.

Keywords: Multiple Sclerosis, IL-12, Gene Polymorphism

\section{Background}

Multiple sclerosis (MS) is a chronic autoimmunemediated demyelinating disorder of the central nervous system (CNS), which has four clinical patterns including relapsing-remitting (RRMS), progressive relapsing (PRMS), primary progressive (PPMS), and secondary progressive (SPMS) (1). The autoreactive CD $4^{+}$helper T (Th) cells, specifically Th1 and Th17, play prominent roles in the pathogenesis of MS and its corresponding animal model, which is recognized as experimental autoimmune encephalomyelitis
(EAE) $(2,3)$. However, the activity of regulatory T (Treg)-and Th2 cells was associated with a reduction in CNS inflammation and improvement of MS and EAE symptoms (4-6), diminished levels of a Th2/Treg-related chemokine (CCL22), unchanged levels of a Treg-related cytokine (IL-35), and elevated concentrations of a Th17-related chemokine (CCL20) in patients with MS $(5,7,8)$.

Both interferon gamma (IFN- $\gamma$ )-producing Th1 cells and interleukin (IL)17-producing Th17 cells contribute in the MS and EAE development $(2,3)$. Th1 cells lead to infiltration of macrophages into the spinal cord, whereas Th17 
cells cause neutrophil infiltration, especially in the brain $(9,10)$. IL-12 may play a major role in the EAE development through the induction of differentiation in both Th1 and Th17 cells (11). IL-12 induces the generation of Th1 cells characterized by the secretion of cytokines such as IFN- $\gamma$, IL-2, and TNF- $\alpha$ (12). IFN- $\gamma$ is a powerful cytokine for upregulation of inducible nitric oxide synthase (NOS) resulting in high levels of NO production by dendritic cells (DCs) and macrophages. Therefore, it is reported that the IL-12/IFN$\gamma / \mathrm{NO}$ axis plays a critical role in the development of EAE, and the level of NOS expression in CNS correlates with EAE severity $(11,13)$. IL-12 also induces the expression of IL-7 in microglia, macrophages, and astrocytes (14). IL-7 is a key cytokine for survival and proliferation of Th1 and Th17 cells in EAE and MS diseases (15).

Structurally, IL-12 consists of two covalently linked P35 and $\mathrm{P} 40$ subunits, which provide a reciprocal connection between the innate and adaptive immunity $(11,16)$. The main sources of IL-12 are antigen-presenting cells such as DCs and macrophages (12). IL-12 receptor is composed of two polypeptide chains, IL- $12 \mathrm{R} \beta 1$ and IL- $12 \mathrm{R} \beta 2$, which is expressed on a number of leukocytes, including natural killer (NK) cells, and T and B lymphocytes (12). The human IL-12B gene is located on chromosome 5q31-33 and encodes the P40 subunit of IL-12 and IL-23 cytokines; hence, it is involved in the induction of IL-12/Th1 cells and IL-23/Th17related immune responses. Based on human gene mutation database (HGMD), 14 SNPs are found in IL-12B gene to date and many studies were focused mainly on a SNP, 1188A $>\mathrm{C}$ (rs3212227), which is located on the 3'-untranslated region of the IL-12B locus (17).

It is also demonstrated that the cytokine production is regulated by genetic parameters in such a way that definite SNPs in cytokine-related genes are associated with high or low cytokine production, which in turn may influence the susceptibility to particular diseases (18). The SNP rs3212227 in $I L-12 B$ gene also influences IL-12 production and is associated with several immune-mediated diseases $(19,20)$. The association of SNP rs3212227 with some autoimmune and inflammatory diseases such as asthma (21), rheumatoid arthritis (RA) (22), inflammatory bowel disease (23), psoriasis (24), ankylosing spondylitis (25) and type 1 diabetes (26) are demonstrated. There are also some investigations on the relationship between SNP rs3212227 and MS, although the results were controversial (27-30). The current study aimed at evaluating the relationship between SNP rs3212227 in IL-12B gene and MS disease in patients from Southeast of Iran.

\section{Methods}

\subsection{Subjects}

Blood samples were collected from 140 patients (46 males and 94 females) with MS referring to Shephah Hospital affiliated to Kerman University of Medical Sciences, Kerman, Southeast of Iran, from January 2014 to February 2015. The expert neurologists confirmed MS diagnosis in the patients, based on the McDonald criteria in clinical and paraclinical evidence (magnetic resonance imaging (MRI) study, oligoclonal bands in CSF, and evoked potentials) (31).

The control group comprised of 140 healthy subjects (47 males and 93 females) recruited from blood donors of Kerman Transfusion Organization. All control subjects were in good general health conditions without a history of CNS disease or other relevant disorders. The Ethical Committee of Kerman University of Medical Sciences evaluated and approved the study protocol. The study objectives and procedure were explained to all participants and they signed the written consent form prior to enrolment in the study. A peripheral blood sample was obtained from all subjects and the genomic DNA was extracted for analysis.

\subsection{DNA Extraction}

The peripheral blood samples were added to EDTA (ethylenediaminetetraacetic acid) pre-treated tubes and then, genomic DNA extraction was performed on the leukocytes by salting out method as previously described by Miller et al. (32). Extracted DNAs were kept at $-20^{\circ} \mathrm{C}$ until use.

\subsection{Polymorphism Genotyping}

The genetic variations of SNP rs3212227 in IL-12 gene was determined by the polymerase chain reaction-restriction length polymorphism (PCR-RFLP) method. The PCR performed in a $25-\mu \mathrm{L}$ reaction mixture containing $12.5 \mu \mathrm{L}$ of PCR master mix (Cinagen, Iran), $1 \mu \mathrm{L}$ of prepared DNA, $2 \mu \mathrm{L}$ of forward primer (5'-TTTGGAGGAAAAGTGGAAGA-3'), $2 \mu \mathrm{L}$ of reverse primer (5'-AACATTCCATACATCCTGGC-3'), stock concentration of $25 \mathrm{ng} / \mu \mathrm{L}$, and $7.5 \mu \mathrm{L}$ of sterile doubledistilled water. The PCR reactions were performed under standard conditions, according to the following program:

One initial phase at $96^{\circ} \mathrm{C}$ for 10 minutes, followed by 30 cycles including denaturation phase at $96^{\circ} \mathrm{C}$ for $30 \mathrm{sec}-$ onds, annealing phase at $61^{\circ} \mathrm{C}$ for 45 seconds, and elongation phase at $72^{\circ} \mathrm{C}$ for 45 seconds. The amplified PCR product was digested with Taq1 restriction enzyme (New England Biolabs, England), which has an exclusive restriction site at SNP rs3212227. In the homozygous situation (AA), a 302-bp fragment (without any digestion); in the heterozygous form (AC), three different fragments of 302, 162-, and 140-bp; and in the homozygous situation (CC), 
two different fragments of 162- and 140-bp were observed. Electrophoresis of the digested PCR final products was performed on a $2.5 \%$ agarose gel, then stained with ethidium bromide and finally studied on a Chemi-Doc model XRS (Bio-Rad, USA) (Figure 1).

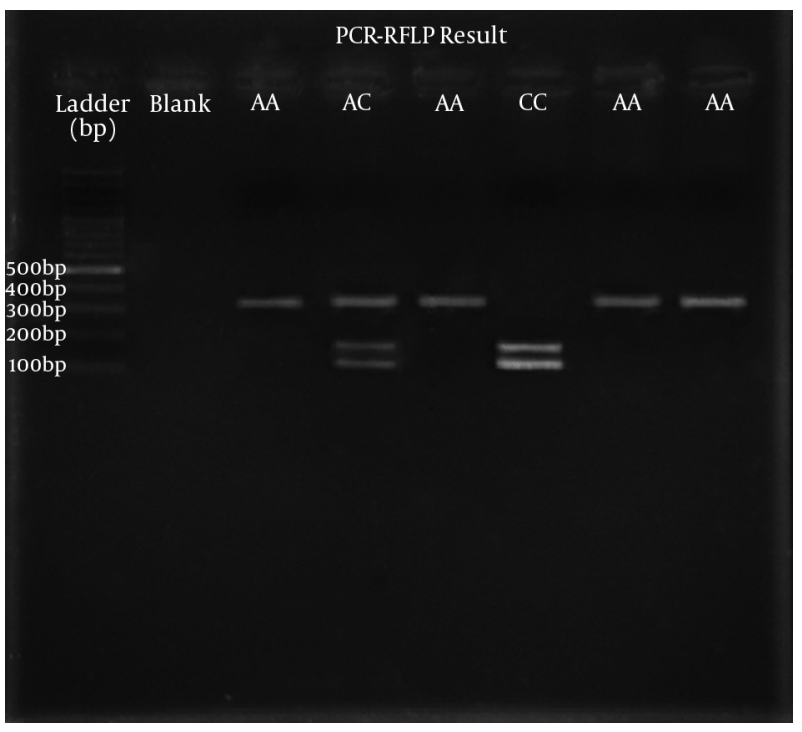

Figure 1. PCR-RFLP shows genetic variation in SNP rs3212227 in IL-12B gene. The column 1 shows the ladder pattern, Column 2 is blank, columns 3, 5, 7, and 8 represent AA homozygous; the column 4 represents AC heterozygous and the column 6 shows CC homozygous.

\subsection{Statistical Analysis}

The $\chi^{2}$ and the Student $\mathrm{t}$ tests were used to compare variables between the groups. The frequency of allele and genotype were measured in patients with MS and healthy controls by direct gene counting. The data were analyzed with SPSS version 18.0 (Chicago, IL, USA); P value < 0.05was considered statistically significant.

\section{Results}

The mean age was $34.98 \pm 8.25$ years in the patients and $36.07 \pm 8.11$ years in the control groups. The gender distribution was 94 (67.1\%) females and 46 (32.9\%) males in the patients and 93 (66.4\%) females and 47 (33.6\%) males in the control groups. There was no significant difference between patients with MS and the healthy controls regarding the age and gender distribution $(\mathrm{P}=0.26$ and $\mathrm{P}=0.89$, respectively). Demographic characteristics of patients with MS such as disease pattern, medication, and disabling symptoms are summarized in Table 1.

\begin{tabular}{|c|c|}
\hline Variable & \\
\hline Age, $y$, mean $\pm S D$ & $34.98 \pm 8.25$ \\
\hline \multicolumn{2}{|l|}{ Gender } \\
\hline Male & $46(32.9 \%)$ \\
\hline Female & $94(67.1 \%)$ \\
\hline \multicolumn{2}{|l|}{ Disease pattern } \\
\hline RRMS & 65 \\
\hline SPMS & 37 \\
\hline PPMS & 19 \\
\hline PRMS & 14 \\
\hline Not recorded & 5 \\
\hline \multicolumn{2}{|l|}{ Medication } \\
\hline Interferon & 55 \\
\hline Methylprednisolone & 15 \\
\hline Methylprednisolone + Interferon & 10 \\
\hline No treatment & 47 \\
\hline Not recorded & 13 \\
\hline \multicolumn{2}{|l|}{ Disabling symptom } \\
\hline Muscle weakness & $88(62.85 \%)$ \\
\hline Muscle spasm & $35(25.00 \%)$ \\
\hline Visual problems & $39(27.85 \%)$ \\
\hline Urinary incontinence & $36(25.71 \%)$ \\
\hline Fatigue & $33(23.57 \%)$ \\
\hline Lack of balance & $41(29.28 \%)$ \\
\hline Dysarthria & $20(14.28 \%)$ \\
\hline Headache & $28(20.00 \%)$ \\
\hline
\end{tabular}

\subsection{MS Patients}

3.1.1. The Association of the Genetic Variations in SNP rs3212227 with MS Disease

The frequency of SNP rs3212227 genotypes in IL-12B gene in patients with MS and controls are summarized in Table 2. The frequency of AA, AC, and CC genotypes were $55.0 \%$, $17.9 \%$, and $27.1 \%$ in patients with MS, and $61.4 \%, 29.3 \%$, and 9.3\% in healthy subjects, respectively. The frequency of CC genotype in SNP rs3212227 was significantly higher in patients with MS compared with the healthy controls $(\mathrm{P}<$ 0.001). However, the prevalence of AC genotype was significantly lower in patients with MS than the healthy controls $(\mathrm{P}<0.02)$. No significant difference was observed between patients with MS and controls regarding the AA genotype frequency (Table 2).

The frequency of A and C alleles in SNP rs3212227 were 63.09\% and $36.07 \%$ in patients with MS, and $76.07 \%$ and $23.9 \%$ in the healthy control subjects, respectively. The frequency of $C$ allele was significantly higher, whereas the frequency of A allele was lower in patients with MS compared with those of the healthy controls $(\mathrm{P}<0.001)$ (Table 2$)$. 
Table 2. The Frequency of Genotypes and Alleles in SNP rs3212227 in IL-12B Gene in Patients with and Controls

\begin{tabular}{lccc}
\hline Genotype & $\begin{array}{c}\text { Patients with MS, N } \\
(\%)\end{array}$ & $\begin{array}{c}\text { Healthy Subjects, } \mathbf{N} \\
(\%)\end{array}$ & P-Value \\
\hline AA & $77(55.0)$ & $86(61.4)$ & 0.27 \\
\hline AC & $25(17.9)$ & $41(29.3)$ & 0.02 \\
\hline CC & $38(27.1)$ & $13(9.3)$ & 0.001 \\
\hline A & $179(63.9)$ & $213(76.07)$ & 0.001 \\
\hline C & $101(36.07)$ & $67(23.9)$ & 0.001 \\
\hline
\end{tabular}

3.1.2. The Association of the Genetic Variations in SNP rs3212227 with Patterns of MS Disease

The frequency of the genotypes and alleles in SNP rs3212227 in patients with MS according to their disease pattern are summarized in Table 3. There were no significant differences between patients with different types of MS forms regarding the frequency of genotypes and alleles in rs3212227. The frequency of CC genotype in SNP rs3212227 was significantly higher in patients with RRMS, SPMS, and PPMS patterns in comparison with the healthy controls ( $\mathrm{P}$ $<0.002, \mathrm{P}<0.001$, and $\mathrm{P}<0.05$, respectively). In patients with RRMS pattern, the frequency of AC genotype (18.6\%) was significantly lower than the healthy controls $(29.3 \%$, $\mathrm{P}<0.05)$. No significant difference was found between SPMS/PPMS and the healthy controls with respect to the frequency of AC genotype in rs3212227, although this parameter was less prevalent in patients with SPMS and PPMS patterns. In RRMS and SPMS patterns, the frequency of patients carrying allele $C$ was significantly higher, whereas the frequency of the ones carrying allele A was significantly lower compared with the healthy controls $(\mathrm{P}<0.03$ and $\mathrm{P}$ $<0.001$, respectively) (Table 3 ).

\section{Discussion}

The results of the present study showed that the frequency of CC genotype and C allele in SNP rs3212227 were significantly higher, whereas the frequency of AC genotype and A allele were lower in patients with MS compared with the healthy controls. The results represent that the presence of CC genotype and C allele in SNP rs3212227 were associated with susceptibility to MS disease, whereas the presence of AC genotype and A allele may confer protection against the disease.

Similarly, the frequency of CC genotype in SNP rs3212227 was significantly higher in patients with RRMS, SPMS, and PPMS patterns in comparison with the healthy controls. In three subgroups of MS, the frequency of AC genotype in the patients was lower than that of the healthy controls, although the parameter was significant for RRMS pattern. Moreover, in all investigated subgroups of patients with MS, the frequency of C allele was more prevalent in the patients than the healthy controls, although it was somehow significant for RRMS and SPMS patterns. It is presumed that using a larger sample size, similar results are obtained for subgroups of patients. Accordingly, the results represented that the presence of CC genotype and C allele in SNP rs3212227 may have equal influences in the susceptibility to three studied MS patterns. On the other hand, the presence of A allele in SNP rs3212227 may confer similar protection against all MS forms.

There are a few studies on the relationship between SNP rs3212227 and MS disease. In accordance with the findings of the current study, the results of a study from Russia showed that the presence of CC genotype in SNP rs3212227 was more prevalent in patients with MS compared with the healthy controls (28). Wang et al., also reported that the frequency of minor allele (C) in SNP rs3212227 of IL-12B gene was significantly higher in patients with RA than the control subjects and patients with RA who possess CC genotype or $\mathrm{C}$ allele, produced significantly higher levels of RF compared with those of the controls (22). Similarly, it is reported that the presence of CC genotype and $\mathrm{C}$ allele in SNP rs3212227 were more prevalent in patients with Behcet disease as compared with the normal controls (33). Moreover, an association was also reported between the presence of CC genotype in SNP rs3212227 and susceptibility to ankylosing spondylitis (25). However, the results of a study showed no association between SNP rs3212227 and MS susceptibility in patients from Southern China (27). No significant association was also reported between SNP rs3212227 and MS in a study from UK (29). The discrepancies may be largely attributed to differences in the age, gender ratio, geographical conditions, socioeconomic status, race, and ethnic background of participants. Furthermore, different inclusion criteria may be accounted for some differences.

The mechanism (s) by which genetic variations in SNP rs3212227 may influence the susceptibility to MS disease remain to be clear in further studies. The SNP rs3212227 may influence the susceptibility to MS through its regulatory effects on IL-12 production. It is demonstrated that the presence of CC genotype or C allele in SNP rs3212227 is associated with higher IL-12 plasma levels in normal controls and patients with Mycobacterium tuberculosis infection (19, 20). The SNP rs3212227 may increase the expression of IL-12 P40; therefore, reinforces the differentiation of naïve T-cells towards Th1/Th17 cells and cause the secretion of a cascade of inflammatory cytokines. However, the SNP rs3212227 may influence IL-12 expression through the effects on the cytokine gene promoter activity, enhancer activity, transcriptional process, and the splicing efficiency. The presence of C allele in SNP rs3212227 may be functional 


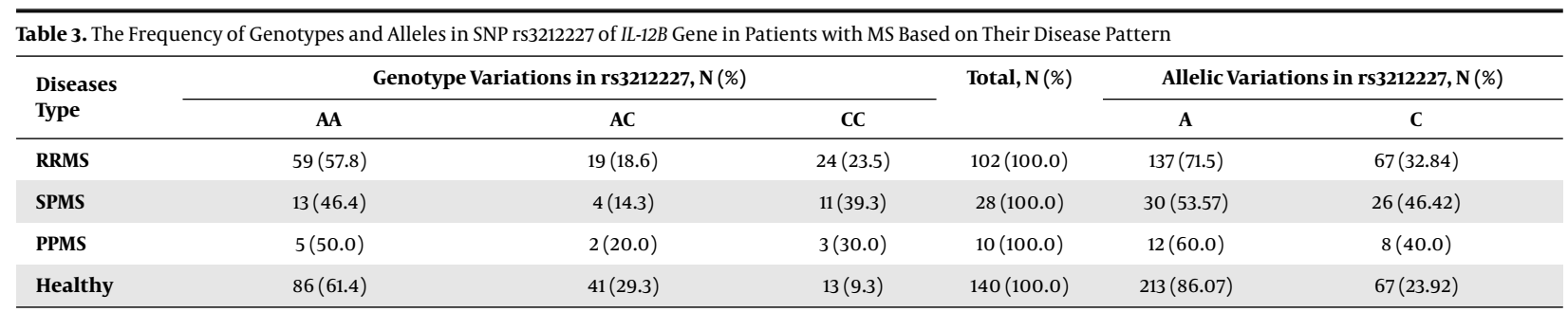

and leads to enhanced IL-12 expression through decreasing the binding of transcriptional repressor, and therefore, increasing the binding affinity of transcription factors. On the other hand, the presence of A allele in SNP rs3212227 may lead to the reduced IL-12 expression through inducing the loss of binding of some transcription factors to the cytokine gene promoter. Accordingly, the presence of $\mathrm{C}$ allele in SNP rs3212227 may lead to the markedly higher IL-12 expression, and therefore, cause the increased Th1 and/or Th17-related immune responses, which contribute to the pathogenesis of MS diseases.

However, several studies found that the presence of C allele in SNP rs3212227 is associated with lower IL-12 P40 production by PBMC following in vitro stimulation (34). The presence of CC/AC genotypes in rs3212227 is also associated with considerable low serum IL-12 P40 levels in osteosarcoma patients (35). There are also other studies, which showed that different genotypes of rs3212227 have no functional effects on IL-12 P40 production (36). These disagreements between different results may be attributed to the differences in ethnicity, disease type, and designs of the studies. It should be noted that in the majority of circumstance, however, it is unclear whether such polymorphisms directly perform the functional effects or are in linkage disequilibrium with another functional SNP in their proximity. Whether the SNP rs3212227 is itself a functional polymorphism that exerts a direct effect on IL-12 expression or whether it is in linkage disequilibrium with secondary functional SNP remains to be clear.

In conclusion, the results of the present study represented that the presence of CC genotype and C allele in SNP rs3212227 were associated with susceptibility to MS disease, whereas the presence of AC genotype and A allele may confer a protection against disease. The CC genotype and $\mathrm{C}$ allele in SNP rs3212227 may have equal influences in the susceptibility to three studied MS patterns. On the other hand, the presence of AC genotype and A allele may confer similar protection against all MS forms.

\section{Footnotes}

Authors' Contributions: Doing tests, acquisition of data, and drafting of the manuscript: Maryam Nemati and Zahra
Etesam, Data gathering: Hossain Hagghani, Selection of patients: Hossain-Ali Ebrahimi, Study concept and design, study supervision, and writing of the manuscript: Abdollah Jafarzadeh. All authors read and approved the final copy of the manuscript.

Funding / Support: The current study was supported by Neurology Research Center, Kerman University of Medical Sciences, Kerman, Iran.

Conflict of Interest: Authors declared no conflict of interest.

\section{References}

1. Confavreux C, Vukusic S. The clinical course of multiple sclerosis. Handb Clin Neurol. 2014;122:343-69. doi: 10.1016/B978-0-444-520012.00014-5. [PubMed: 24507525].

2. Ghaffari SA, Nemati M, Hajghani H, Ebrahimi H, Sheikhi A, Jafarzadeh A. Circulating concentrations of interleukin (IL)-17 in patients with multiple sclerosis: Evaluation of the effects of gender, treatment, disease patterns and IL-23 receptor gene polymorphisms. Iran J Neurol. 2017;16(1):15-25. [PubMed: 28717429]. [PubMed Central: PMC5506751].

3. Jafarzadeh A, Azizi SV, Nemati M, Khoramdel-Azad H, Shamsizadeh A, Ayoobi F, et al. Ginger Extract Reduces the Expression of IL-17 and IL23 in the Sera and Central Nervous System of EAE Mice. Iran J Immunol. 2015;12(4):288-301. [PubMed: 26714420].

4. Etesam Z, Nemati M, Ebrahimizadeh MA, Ebrahimi HA, Hajghani H, Khalili T, et al. Altered Expression of Specific Transcription Factors of Th17 (RORgammat, RORalpha) and Treg Lymphocytes (FOXP3) by Peripheral Blood Mononuclear Cells from Patients with Multiple Sclerosis. J Mol Neurosci. 2016;60(1):94-101. doi: 10.1007/s12031-016-0789-5. [PubMed: 27370539].

5. Jafarzadeh A, Jamali M, Mahdavi R, Ebrahimi HA, Hajghani H, Khosravimashizi A, et al. Circulating levels of interleukin-35 in patients with multiple sclerosis: evaluation of the influences of FOXP3 gene polymorphism and treatment program. J Mol Neurosci. 2015;55(4):891-7. doi: 10.1007/s12031-014-0443-z. [PubMed: 25326790].

6. Jafarzadeh A, Mahdavi R, Jamali M, Hajghani H, Nemati M, Ebrahimi HA. Increased Concentrations of Interleukin-33 in the Serum and Cerebrospinal Fluid of Patients with Multiple Sclerosis. Oman Med J. 2016;31(1):40-5. doi: 10.5001/omj.2016.08. [PubMed: 26813806]. [PubMed Central: PMC4720939].

7. Jafarzadeh A, Ebrahimi HA, Bagherzadeh S, Zarkesh F, Iranmanesh F, Najafzadeh A, et al. Lower serum levels of Th2-related chemokine CCL22 in women patients with multiple sclerosis: a comparison between patients and healthy women. Inflammation. 2014;37(2):604-10. doi: 10.1007/s10753-013-9775-z. [PubMed: 24254331].

8. Jafarzadeh A, Bagherzadeh S, Ebrahimi HA, Hajghani H, Bazrafshani MR, Khosravimashizi A, et al. Higher circulating levels of chemokine CCL20 in patients with multiple sclerosis: evaluation of the influences of chemokine gene polymorphism, gender, treatment and dis- 
ease pattern. J Mol Neurosci. 2014;53(3):500-5. doi: 10.1007/s12031-0130214-2. [PubMed: 24395091].

9. Kroenke MA, Carlson TJ, Andjelkovic AV, Segal BM. IL-12- and IL-23modulated T cells induce distinct types of EAE based on histology, CNS chemokine profile, and response to cytokine inhibition. J Exp Med. 2008;205(7):1535-41. doi: 10.1084/jem.20080159. [PubMed: 18573909]. [PubMed Central: PMC2442630].

10. Fletcher JM, Lalor SJ, Sweeney CM, Tubridy N, Mills KH. T cells in multiple sclerosis and experimental autoimmune encephalomyelitis. Clin Exp Immunol. 2010;162(1):1-11. doi: 10.1111/j.1365-2249.2010.04143.x. [PubMed: 20682002]. [PubMed Central: PMC2990924].

11. Jafarzadeh A, Ahangar-Parvin R, Nemat M, Taghipour Z, Shamsizadeh A, Ayoobi F, et al. Ginger extract modulates the expression of IL-12 and TGF-beta in the central nervous system and serum of mice with experimental autoimmune encephalomyelitis. Avicenna J Phytomed. 2017;7(1):54-65. [PubMed: 28265547]. [PubMed Central: PMC5329177].

12. Lasek W, Zagozdzon R, Jakobisiak M. Interleukin 12: still a promising candidate for tumor immunotherapy? Cancer Immunol Immunother. 2014;63(5):419-35. [PubMed: 24514955]. [PubMed Central: PMC3994286].

13. Xiao BG, Ma CG, Xu LY, Link H, Lu CZ. IL-12/IFN-gamma/NO axis plays critical role in development of Th1-mediated experimental autoimmune encephalomyelitis. Mol Immunol. 2008;45(4):1191-6. doi: 10.1016/j.molimm.2007.07.003. [PubMed: 17697713].

14. Jana M, Mondal S, Jana A, Pahan K. Interleukin-12 (IL-12), but not IL23, induces the expression of IL-7 in microglia and macrophages: implications for multiple sclerosis. Immunology. 2014;141(4):54963. doi: 10.1111/imm.12214. [PubMed: 24224652]. [PubMed Central: PMC3956429].

15. Lee LF, Axtell R, Tu GH, Logronio K, Dilley J, Yu J, et al. IL-7 promotes $\mathrm{T}(\mathrm{H}) \mathbf{1}$ development and serum IL-7 predicts clinical response to interferon-beta in multiple sclerosis. Sci Transl Med. 2011;3(93):93ra68. doi: 10.1126/scitranslmed.3002400. [PubMed: 21795588]. [PubMed Central: PMC3739690].

16. Croxford AL, Kulig P, Becher B. IL-12-and IL-23 in health and disease. Cytokine Growth Factor Rev. 2014;25(4):415-21. doi: 10.1016/j.cytogfr.2014.07.017. [PubMed: 25130295].

17. Zhou L, Yao F, Luan H, Wang Y, Dong X, Zhou W, et al. Functional polymorphisms in the interleukin-12 gene contribute to cancer risk: evidence from a meta-analysis of 18 case-control studies. Gene. 2012;510(1):71-7. doi:10.1016/j.gene.2012.08.019. [PubMed: 22940148].

18. Tahmasebi Z, Akbarian M, Mirkazemi S, Shahlaee A, Alizadeh Z, Amirzargar AA, et al. Interleukin-1 gene cluster and IL-1 receptor polymorphisms in Iranian patients with systemic lupus erythematosus. Rheumatol Int. 2013;33(10):2591-6. doi: 10.1007/s00296-013-2784-2. [PubMed: 23722873].

19. Peresi E, Oliveira LR, da Silva WL, da Costa EA, Araujo JJ, Ayres JA, et al. Cytokine Polymorphisms, Their Influence and Levels in Brazilian Patients with Pulmonary Tuberculosis during Antituberculosis Treatment. Tuberc Res Treat. 2013;2013:285094. doi: 10.1155/2013/285094. [PubMed: 23634300]. [PubMed Central: PMC3619634].

20. Selvaraj P, Alagarasu K, Harishankar M, Vidyarani M, Nisha Rajeswari D, Narayanan PR. Cytokine gene polymorphisms and cytokine levels in pulmonary tuberculosis. Cytokine. 2008;43(1):26-33. doi: 10.1016/j.cyto.2008.04.011. [PubMed: 18522869].

21. Chen T, Liang W, Gao L, Wang Y, Liu Y, Zhang L, et al. Association of single nucleotide polymorphisms in interleukin 12 (IL-12A and -B) with asthma in a Chinese population. Hum Immunol. 2011;72(7):603-6. doi: 10.1016/j.humimm.2011.03.018. [PubMed: 21513752].

22. Wang EY, Yang Q, Liao ZG. Association of polymorphisms in inter- leukin (IL)-12A and -B genes with rheumatoid arthritis in a Chinese population. Clin Exp Immunol. 2015;180(1):83-9. doi: 10.1111/cei.12563. [PubMed: 25469793]. [PubMed Central: PMC4367096].

23. Lopez-Hernandez R, Valdes M, Campillo JA, Martinez-Garcia P, Salama $\mathrm{H}$, Bolarin JM, et al. Pro- and anti-inflammatory cytokine gene singlenucleotide polymorphisms in inflammatory bowel disease. Int J Immunogenet. 2015;42(1):38-45. [PubMed: 25359546].

24. $\mathrm{Li} \mathrm{XL}, \mathrm{Wu} \mathrm{CF}, \mathrm{Wu}$ GS. Genetic variations of cytokines and cytokine receptors in psoriasis patients from china. Int J Genomics. 2014;2014:870597.

25. Wong RH, Wei JC, Huang CH, Lee HS, Chiou SY, Lin SH, et al. Association of IL-12B genetic polymorphism with the susceptibility and disease severity of ankylosing spondylitis.J Rheumatol. 2012;39(1):135-40 doi: 10.3899/jrheum.110613. [PubMed: 22045842].

26. Eiris N, Gonzalez-Lara L, Santos-Juanes J, Queiro R, Coto E, Coto-Segura P. Genetic variation at IL12B, IL23R and IL23A is associated with psoriasis severity, psoriatic arthritis and type 2 diabetes mellitus. J Dermatol Sci. 2014;75(3):167-72. doi:10.1016/j.jdermsci.2014.05.010. [PubMed: 24957500].

27. Liu M, Hu X, Wang Y, Chen X, Wu J. Association of IL-23 and its receptor gene single-nucleotide polymorphisms with multiple sclerosis in Chinese southern population. Int J Neurosci. 2014;124(12):904-7. doi: 10.3109/00207454.2014.894044. [PubMed: 24547735].

28. Alifirova VM, Orlova I, Babenko SA, Rudko AA, Puzyrev VP. [The 1188 A/C ILI2B gene polymorphism in patients with multiple sclerosis and in healthy subjects of Tomsk region]. Vserossiiskoe Obshchestvo Psikhiat. 2006;3:130-5.

29. Hall MA, McGlinn E, Coakley G, Fisher SA, Boki K, Middleton D, et al. Genetic polymorphism of IL-12 p40 gene in immune-mediated disease. Genes Immun. 2000;1(3):219-24. doi: 10.1038/sj.gene.6363661. [PubMed: 11196715].

30. Huang J, Yang Y, Zhou F, Liang Z, Kang M, Kuang Y, et al. Metaanalysis of the IL23R and IL12B polymorphisms in multiple sclerosis. Int J Neurosci. 2016;126(3):205-12. doi:10.3109/00207454.2015.1007508. [PubMed: 26000455].

31. Polman $\mathrm{CH}$, Reingold SC, Banwell B, Clanet $\mathrm{M}$, Cohen JA, Filippi $M$, et al. Diagnostic criteria for multiple sclerosis: 2010 revisions to the McDonald criteria. Ann Neurol. 2011;69(2):292-302. doi: 10.1002/ana.22366. [PubMed: 21387374]. [PubMed Central: PMC3084507].

32. Miller SA, Dykes DD, Polesky HF. A simple salting out procedure for extracting DNA from human nucleated cells. Nucleic Acids Res. 1988;16(3):1215. [PubMed: 3344216]. [PubMed Central: PMC334765].

33. Li X, Bai L, Fang J, Hou S, Zhou Q, Yu H, et al. Genetic variations of IL-12B, IL-12Rbeta1, IL-12Rbeta2 in Behcet's disease and VKH syndrome. PLoS One. 2014;9(5). e98373. doi: 10.1371/journal.pone.0098373. [PubMed: 24859272]. [PubMed Central: PMC4032269].

34. Stanilova S, Miteva L. Taq-I polymorphism in 3'UTR of the IL-12B and association with IL-12p40 production from human PBMC. Genes Immun. 2005;6(4):364-6. doi: 10.1038/sj.gene.6364213. [PubMed: 15858599].

35. Wang J, Nong L, Wei Y, Qin S, Zhou Y, Tang Y. Association of interleukin12 polymorphisms and serum IL-12p40 levels with osteosarcoma risk. DNA Cell Biol. 2013;32(10):605-10. doi: 10.1089/dna.2013.2098. [PubMed: 23991654].

36. Ong'echa JM, Raballah EO, Kempaiah PM, Anyona SB, Were T, Davenport GC, et al. Polymorphic variability in the 3' untranslated region (UTR) of IL12B is associated with susceptibility to severe anaemia in Kenyan children with acute Plasmodium falciparum malaria. BMC Genet. 2011;12:69. doi: 10.1186/1471-2156-12-69. [PubMed: 21819616] [PubMed Central: PMC3166270]. 